\title{
Partition Polynomials: Asymptotics and Zeros
}

\author{
Robert P. Boyer, William M. Y. Goh
}

January 19, 2021

\begin{abstract}
Let $F_{n}(x)$ be the partition polynomial $\sum_{k=1}^{n} p_{k}(n) x^{k}$ where $p_{k}(n)$ is the number of partitions of $n$ with $k$ parts. We emphasize the computational experiments using degrees up to 70,000 to discover the asymptotics of these polynomials. Surprisingly, the asymptotics of $F_{n}(x)$ have two scales of orders $n$ and $\sqrt{n}$ and in three different regimes inside the unit disk. Consequently, the zeros converge to network of curves inside the unit disk given in terms of the dilogarithm.
\end{abstract}

\section{Introduction}

Let $p(n)$ denote the number of partitions of a positive integer $n$; that is, the number of ways of writing $n$ additively. Euler identified their generating function:

$$
P(u)=\prod_{k=1}^{\infty} \frac{1}{1-u^{k}}=\sum_{k=1}^{\infty} p(n) u^{k}
$$

By inverting this equation, we find an integral form for $p(n)$ as

$$
p(n)=\frac{1}{2 \pi i} \oint_{|u|=r} \frac{P(u)}{u^{n+1}} d u, \quad 0<r<1 .
$$

In their celebrated work, Hardy and Ramanujan in 1917 [6] discovered the asymptotics of $p(n)$ by developing a new approach "the circle method" to handle the asymptotics of such contour integrals which have dense singularities over the unit circumference. A basic form of their asymptotics is

$$
p(n)=\frac{1}{4 n \sqrt{3}} e^{\pi \sqrt{2 n / 3}}(1+O(1 / \sqrt{n}))
$$

We emphasize that Hardy and Ramanujan were guided by an extensive list that appears in their paper of values of $p(n)$ up to $n=200$.

The partition polynomial $F_{n}(x)$ is a refinement of the partition numbers:

$$
F_{n}(x)=\sum_{k=1}^{n} p_{k}(n) x^{k}
$$

where $p_{k}(n)$ is the number of partitions of $n$ with exactly $k$ parts. For example, $F_{5}(x)=x^{5}+x^{4}+2 x^{3}+2 x^{2}+$ $x$. We note that the partition numbers are recovered as $F_{n}(1)=p(n)$. Further, $p_{k}(n)$ can be computed via the recurrence $p_{k}(n)=p_{k-1}(n-1)+p_{k}(n-k)$. 

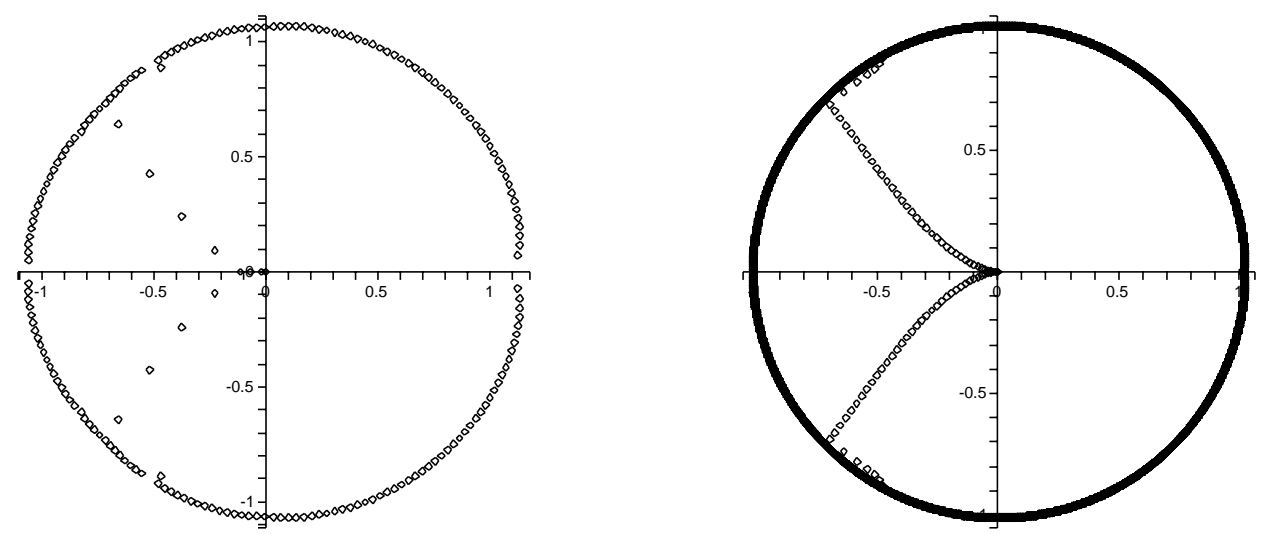

Figure 1: (a) Partition Polynomial Zeros for Degree 200; $\quad$ (b) for Degree 10,000

Richard Stanley plotted the zeros of $F_{200}$ and asked what happens as their degrees go to infinity. See Figure 1(a).

Since the zeros of $F_{n}$ are symmetric about the real axis, many times we restricted our attention to the upper half-plane.

We single out many intriguing features of this plot:

1. The zeros are clustering about the unit circle;

2. There is a sparse of zeros in the left half plane;

3. There are gaps near the angle $2 \pi / 3$ and at $x=1$ and $x=-1$.

4. There may be two scales of zeros for those either outside or inside the unit circle;

5. There are $O(n)$ zeros outside the unit circle and $O(\sqrt{n})$ inside. We use $\sqrt{n}$ rather than other powers of $n$ by inspiration from the Hardy-Ramanujan asymptotics from $p(n)$.

6. Empirically we expect the order of convergence of the zeros to be $O(1 / \sqrt{n})[11]$.

We compare these proposed features with the computed zeros of $F_{10000}$ in Figure 1(b). At degree 10,000 all three gaps now disappear while a second family of zeros inside the unit disk appears. This is especially noteworthy at $x=1$ since $F_{n}(1)=p(n)$. Furthermore, the order $O(\sqrt{n})$ of zeros is confirmed inside the disk.

These zeros were found using the MPSolve program described in [3] which is our major tool to obtain the zeros of high degree polynomials. Its underlying algorithm is based on simultaneous approximation of all the zeros and uses the Aberth iteration. One advantage of MPSolve is that it handles integer coefficients with arbitrary large precision. This ability is critical since the coefficients of the partition polynomials have hundreds of digits; for example, in Figure 2(b) there is a plot of the number of digits of the 25,000 degree partition polynomial. Note that the maximal coefficient 168 digits and that the polynomial is unimodal. For comparison in Figure 2(a), we included $F_{500}$ whose maximal number of digits is 19.

In working with such polynomials, a natural question is how to test whether the zeros are relatively correct. The zeros obtained have, say, 20 digits, yet the coefficients of the polynomial have hundreds of 

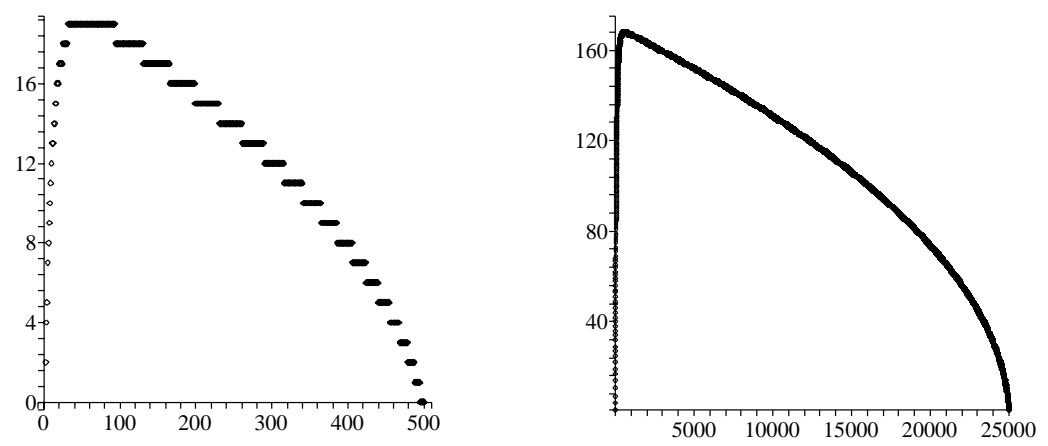

Figure 2: (a) Digits of the Partition Polynomials for Degree 500;

(b) for Degree 25,000

integer coefficients. So there is no possibility of ever checking directly that the zeros through evaluation. On the other hand, the zeros do pass a simple numerical test. We observe that the sum of the zeros equals the negative of the coefficient of $x^{n-1}$ in $F_{n}(x)$ which is always 1 .

The sum of the computed zeros are: at degree 6000, -1.0000000000000000014085 ; at degree 10,000, -0.9999999999999999860563 ; at degree $15,000,-1.00000000000000002067132+2 \times 10^{-19} i$; and at degree 20,000,-1.00000000000000002323212. For $F_{n}$, the coefficient of $x^{n-2}$ is 2 and equals the second elementary symmetric function of the zeros. At degree 6000, for the computed zeros this evaluates to $1.99999999999999999377164+5.62 \times 10^{-26} i$.

Furthermore, the zeros that MPSolve produces coincide extremely well with the asymptotics we discovered so we have much confidence in the computation. In our work there was a complementary interplay between developing the asymptotics and numerically determining their limiting behavior and their densities.

The appealing question of determining the limiting behavior of the zeros is intimately connected with the analytic problem of finding asymptotic formulas for these polynomials as their degrees go to infinity.

\section{Introduction to the Zero Attractor}

We now formalize what we mean by the convergence of the zeros. Let $Z\left(F_{n}\right)$ denote the finite set of zeros of the polynomial $F_{n}$. Then the zero attractor $\mathcal{A}$ of the polynomial sequence $\left\{F_{n}\right\}$ is the limit of $Z\left(F_{n}\right)$ in the Hausdorff metric on the compact subsets of $\mathbb{C}$. In Figure 3(a), there is the plot of the full zero attractor as well as a closeup of the upper left-hand quarter of the attractor in Figure 3(b). We will describe the curves in these plots in Section 4

It may be hard to see but there are actually three curves that make up the zero attractor in the upper half plane. Furthermore the common intersection point of these curves we call the triple point $T$. Its polar coordinates are $r=0.9783370882, \theta=2.356797156$ and its rectangular coordinates are $x=-0.6922055811$ and $y=0.6913717463$.

The zeros converge very slowly to this special point. In Figure 4, there are plots of the zeros near the triple point $T$ for degrees 400, 5,000, and 50,000:

In Table 1, we give the total number of all zeros for $F_{n}$ inside the unit disk which empirically confirms that their order inside the disk is $O(\sqrt{n})$. Its first entry is the count of all zeros inside the unit disk. The second entry is the count of all zeros that lie in $Q_{2}$, the closed quarter unit in the second quadrant excluding 


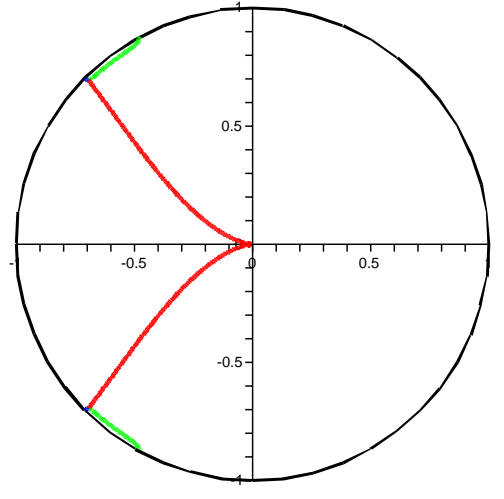

Figure 3: (a) Full Zero Attractor;

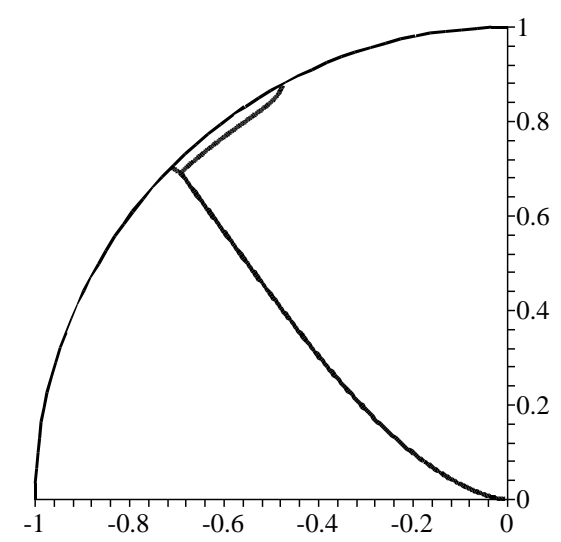

(b) Zero Attractor in Left Half Plane
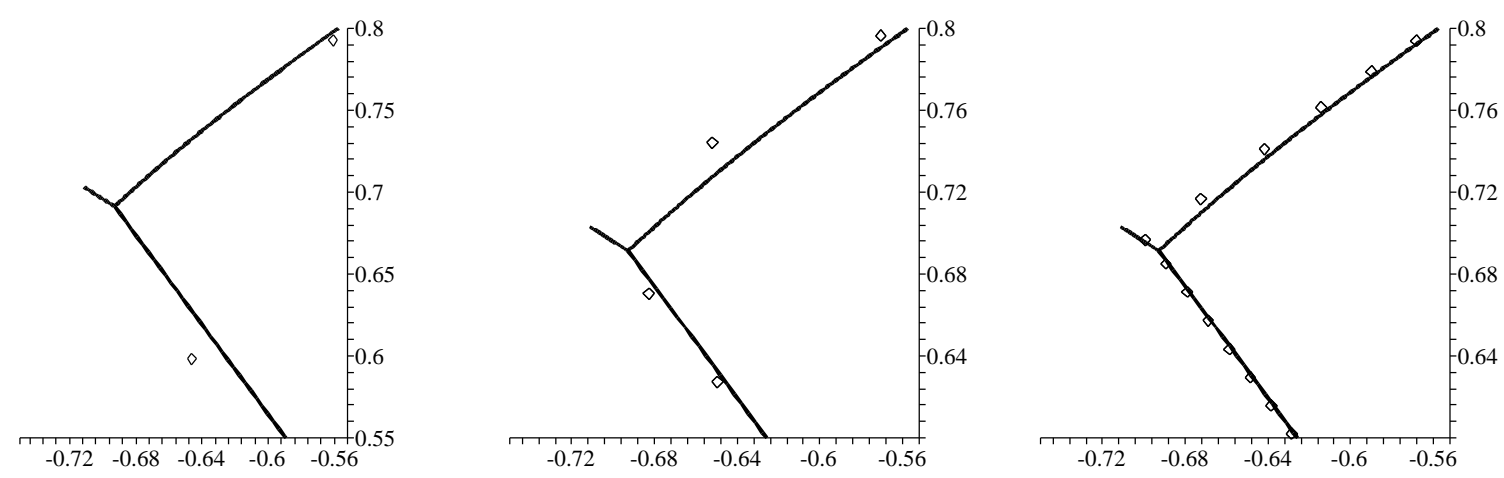

Figure 4: Zeros for Degrees 400, 5,000, and 50,000 Near the Triple Point $T$

the unit circle. The next entries are the counts for the three families of zeros near the three curves seen in Figure 3 that in the second quadrant. These families will be identified in Section 4 , for now, we call them either Family 1, 2, or 3. Note that Family 1 includes zeros that lie along the real axis.

The predicted number of zeros in Table 1 is given by the empirical formula

$$
\text { \# Zeros inside the unit disk } \sim 0.9154 \sqrt{n}
$$

obtained by fitting a multiple of the power law $\sqrt{n}$ to the zeros to the first four polynomials at degrees $5000,10,000,15,000$, and 20,000. Another estimate for these zeros is given in equation (4).

\section{Potential Theory, Statistical Mechanics, and Limits of Zeros}

If $\mu$ is a finite measure with compact support on $\mathbb{C}$, then its logarithmic potential function $U^{\mu}(z)$ is given as

$$
U^{\mu}(z)=\int_{\mathbb{C}} \log \frac{1}{|z-t|} d \mu(t) .
$$


Table 1: Number of Zeros Inside the Unit Disk

\begin{tabular}{|r||c|c|c|c|c||r|}
\hline Degree & \# Zeros & All Zeros in $Q_{2}$ & Family 1 & Family 2 & Family 3 & Prediction \\
\hline 5000 & 64 & 36 & 32 & 4 & 0 & 64.8 \\
10000 & 92 & 51 & 45 & 6 & 0 & 91.7 \\
15000 & 112 & 61 & 53 & 7 & 1 & 112.2 \\
20000 & 130 & 71 & 62 & 8 & 1 & 129.6 \\
25000 & 146 & 79 & 69 & 9 & 1 & 144.9 \\
30000 & 160 & 87 & 76 & 10 & 1 & 158.7 \\
35000 & 172 & 93 & 81 & 11 & 1 & 171.5 \\
40000 & 184 & 99 & 86 & 12 & 1 & 183.3 \\
50000 & 204 & 109 & 95 & 13 & 1 & 204.9 \\
60000 & 226 & 121 & 104 & 15 & 2 & 224.5 \\
70000 & 242 & 129 & 112 & 16 & 1 & 242.5 \\
\hline
\end{tabular}

The connection with polynomial zeros comes from the fact that if $a_{1}, \cdots, a_{n} \in \mathbb{C}$ and $\mu=\frac{1}{n} \sum_{k=1}^{n} \delta_{a_{k}}$, then $U^{\mu}(z)=\frac{1}{n} \ln |P(z)|$, where $P(z)=\left(z-a_{1}\right) \cdots\left(z-a_{n}\right)$.

The hope is that the logarithmic potentials for a sequence of polynomials will converge to the density measure on the zero attractor. Classically, one studies the first limit below while we need the following second limit below as well because the number of zeros inside the unit disk is $O(\sqrt{n})$ :

$$
\lim _{n \rightarrow \infty} \frac{\ln \left[F_{n}(x)\right]}{n}, \quad \lim _{n \rightarrow \infty} \frac{\ln \left[F_{n}(x)\right]}{\sqrt{n}} .
$$

From the Yang-Lee theory of phase transitions in statistical mechanics, the limit of $\ln \left[F_{n}(x)\right] / n$ gives the complex free energy and its zeros indicate the presence of phase transitions.

We found Alan Sokal's formulation [9] to be very helpful because it allows more general normalizations than $n$. Here is his result:

Theorem 1 [Sokal] Let $D$ be a domain in $\mathbb{C}$, and let $z_{0} \in D$. Let $\left\{g_{n}\right\}$ be a sequence of analytic functions in $D$, and let $\left\{a_{n}\right\}$ be a sequence of positive reals such that $\left\{\left.\left|g_{n}\right|\right|_{n}\right\}$ are uniformly bounded on the compact subsets of D. Suppose there does not exist a neighborhood $V$ of $z_{0}$ and a function $v$ on $V$ that is either harmonic or else identically $-\infty$ such that

$$
\liminf _{n \rightarrow \infty} a_{n} \ln \left|g_{n}(x)\right| \leq v(x) \limsup _{n \rightarrow \infty} a_{n} \ln \left|g_{n}(x)\right|
$$

for all $z \in V$. Then $z_{0}$ lies in $\liminf z\left(g_{n}\right)$; that is, for all $n$ sufficiently large, there exists zeros $z_{n}^{*}$ of $g_{n}$ with $\lim _{n \rightarrow \infty} z_{n}^{*}=z_{0}$.

Below we will state what the limits in equation (2) are and see that they determine on three regions inside the disk. This theorem tells us that the zeros accumulate on the boundaries of these regions. Note: these limits are very difficult to compute. 


\section{Explicit Description of Zero Attractor}

Let $\operatorname{Li}_{2}(x)$ denote the dilogarithm of $x$; that is, $\operatorname{Li}_{2}(x)=\sum_{n=1}^{\infty} \frac{x^{n}}{n^{2}}=-\int_{0}^{x} \frac{\ln (1-t)}{t} d t$. We introduce a related family of (sub)harmonic functions

$$
f_{1}(x)=\mathfrak{R}\left[\sqrt{\mathrm{Li}_{2}(x)}\right], \quad f_{k}(x)=\frac{1}{k} \Re\left[\sqrt{\operatorname{Li}_{2}\left(x^{k}\right)}\right]
$$

which are harmonic in different sectors of the unit disk. These functions play a crucial role in the asymptotics of the partition polynomials. Next we introduce the curves $C_{k, \ell}$ inside the unit disk where $f_{k}(x)=f_{\ell}(x)$ which we will informally call $\sqrt{\mathrm{Li}_{2}}$-curves. The zero attractor consists of portions of $C_{12}, C_{13}$, and $C_{23}$.

We can now describe how the plots of the zero attractor are done. Since the $\sqrt{\mathrm{Li}_{2}}$-curves $C_{k, \ell}$ are level curves of the real part of a harmonic function, these curves are naturally solutions to an initial value problem. For convenience, write $L_{k}(z)=\sqrt{\mathrm{Li}_{2}\left(z^{k}\right)} / k$ and $L_{k}(z)^{\prime}$ is just its usual derivative. Then

$$
\frac{d y}{d x}=\frac{\Re\left[L_{k}(x+i y)^{\prime}-L_{\ell}(x+i y)^{\prime}\right]}{\mathfrak{I}\left[L_{k}(x+i y)^{\prime}-L_{\ell}(x+i y)^{\prime}\right]}, \quad y\left(x_{0}\right)=y_{0} .
$$

The initial condition needs to be found numerically; typically, by a value on the unit circle where $\operatorname{Li}_{2}\left(e^{i t}\right)=$ $u(t)+i v(t)$ is known in closed form:

$$
u(t)=\sum_{n=1}^{\infty} \frac{\cos n t}{n^{2}}=\frac{3 t^{2}-6 t \pi+2 \pi^{2}}{12}, \quad v(\theta)=\sum_{n=1}^{\infty} \frac{\sin n t}{n^{2}}=-\int_{0}^{t} \ln \left(2 \sin \frac{\xi}{2}\right) d \xi, \quad 0 \leq t \leq 2 \pi .
$$

Since the $\sqrt{\mathrm{Li}_{2}}$-curves are very smooth and even Euler's method gives an useful plot for them.

In establishing the role of the $\sqrt{\mathrm{Li}_{2}}$-curves for the zero attractor, though, it is better to treat them as boundaries for regions of majorization among the functions $f_{k}$ inside the upper unit disk. We define

$$
\mathcal{R}(1)=\left\{x: f_{1}(x) \geq f_{2}(x), f_{3}(x)\right\}, \mathcal{R}(2)=\left\{x: f_{2}(x) \geq f_{1}(x), f_{3}(x)\right\}, \mathcal{R}(3)=\left\{x: f_{3}(x) \geq f_{1}(x), f_{2}(x)\right\} .
$$

Then $\partial \mathcal{R}(1)$ consists of portions of the unit circle, $C_{12}, C_{13}$, and $[0,1] ; \partial \mathcal{R}(2)$ consists of portions of the unit circle, $C_{12}, C_{23}$, and $[-1,0] ; \partial \mathcal{R}(3)$ consists of portions of the unit circle, $C_{13}$, and $C_{23}$.

The realization that the zeros accumulation on the boundary curves made us examine the majorization among $f_{1}, f_{2}$, and $f_{3}$ on the unit circle. Since these are known in closed form, we found that $f_{1}\left(e^{i t}\right)$ dominates on the $\operatorname{arc} t \in\left[0, \theta_{13}\right], f_{3}\left(e^{i t}\right)$ dominates on $t \in\left[\theta_{13}, \theta_{12}\right]$, and $f_{2}\left(e^{i t}\right)$ dominates on $t \in\left[\theta_{12}, \pi\right]$ where $\theta_{13}=$ $2.066729664<2 \pi / 3=2.094395103, \theta_{12}=2.2536266<3 \pi / 4=2.356194490$, and $\theta_{23}=2.361704176>$ $3 \pi / 4$. The following Theorem together with Sokal's result guarantees that there are no further zero families.

Theorem 2 (a) On the unit disk, $f_{k}(x) \leq \max \left[f_{1}(x), f_{2}(x), f_{3}(x)\right]$, for all $k \geq 4$.

(b) For $x \in \mathcal{R}(1), f_{1}(x) \geq f_{k}(x)$, for all $k \geq 2$.

(c) For $x \in \mathcal{R}(2), f_{2}(x) \geq f_{k}(x)$, for all $k \geq 3$ and $k=1$.

(d) For $x \in \mathcal{R}(3), f_{3}(x) \geq f_{k}(x)$, for all $k \geq 4$ and for $k=1,2$.

The proof is intricate and lengthy and requires subharmonic function theory and special properties of conformal maps. The theorem, though, is easy to verify numerically in special cases.

We next record the values of the normalized limits of $\ln \left|F_{n}(z)\right|$.

Theorem 3 Outside the unit disk, $\lim _{n \rightarrow \infty} \frac{\ln \left[F_{n}(z)\right]}{n}=\ln z, \lim _{n \rightarrow \infty} \frac{\ln \left|F_{n}(z)\right|}{n}=\ln |z|$.

Inside the unit disk, $\lim _{n \rightarrow \infty} \frac{\ln \left[F_{n}(z)\right]}{n}=0$ and $\lim _{n \rightarrow \infty} \frac{\ln \left|F_{n}(z)\right|}{n}=0$. 

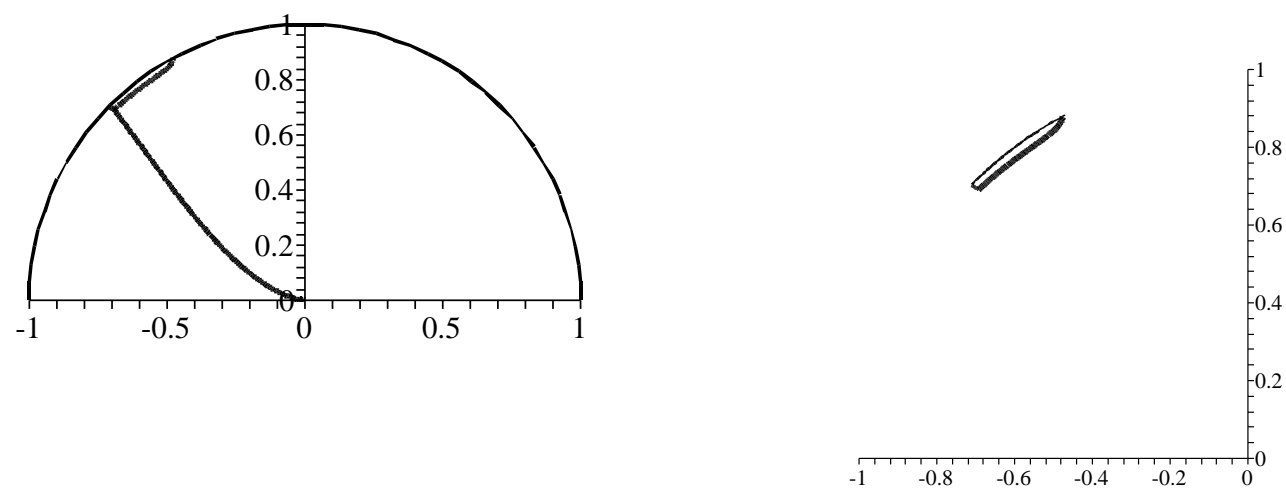

Figure 5: (a) The Three Regions $\mathcal{R}(1), \mathcal{R}(2), \mathcal{R}(3)$; (b) Region $\mathcal{R}(3)$ Separately

By Sokal's theorem, we now know that the $O(n)$ contribution to the zero attractor is the unit circle. The delicate asymptotic expansions needed to establish the following theorem are outlined in Section 6

Theorem 4 1. On region $\mathcal{R}(1), \lim _{n \rightarrow \infty} \frac{\ln \left[F_{n}(z)\right]}{2 \sqrt{n}}=\sqrt{\operatorname{Li}_{2}(z)}$ and $\lim _{n \rightarrow \infty} \frac{\ln \left|F_{n}(z)\right|}{2 \sqrt{n}}=\Re \sqrt{\operatorname{Li}_{2}(z)}$.

2. On region $\mathcal{R}(2), \lim _{n \rightarrow \infty} \frac{\ln \left[F_{n}(z)\right]}{2 \sqrt{n}}=\frac{1}{2} \sqrt{\operatorname{Li}_{2}\left(z^{2}\right)}$ and $\lim _{n \rightarrow \infty} \frac{\ln \left|F_{n}(z)\right|}{2 \sqrt{n}}=\frac{1}{2} \Re \sqrt{\operatorname{Li}_{2}\left(z^{2}\right)}$.

3. On region $\mathcal{R}(3), \lim _{n \rightarrow \infty} \frac{\ln \left[F_{n}(z)\right]}{2 \sqrt{n}}=\frac{1}{3} \sqrt{\operatorname{Li}_{2}\left(z^{3}\right)}$ and $\lim _{n \rightarrow \infty} \frac{\ln \left|F_{n}(z)\right|}{2 \sqrt{n}}=\frac{1}{3} \Re \sqrt{\operatorname{Li}_{2}\left(z^{3}\right)}$.

The above theorem shows that while the limit $\frac{\ln \left|F_{n}(z)\right|}{2 \sqrt{n}}$ is continuous on the unit disk the derivative of the limit fails to be continuous exactly on the boundaries of the three regions. Hence we have determined the zero attractor inside the unit disk:

Theorem 5 The zero attractor for the partition polynomials consists exactly of the unit circle together with the boundaries of the three regions $\mathcal{R}(1), \mathcal{R}(2)$, and $\mathcal{R}(3)$ inside the unit disk.

\section{Zero Density}

The zero densities are determined by our following theorem:

Theorem 6 Density Theorem Let $G$ be a conformal map from a neighborhood of an analytic arc $C$ to a neighborhood sector $S$ of an arc of the unit circle such that $G(C)=S$. Let $\left\{T_{n}(x)\right\}$ be a sequence of analytic functions on $G^{-1}(S)$ such that

$$
T_{n}(x)=1+a_{n}(x) G^{c_{n}}(x)+e_{n}(x)
$$


Table 2: Properties of the Boundary Curves $C_{12} \cap \mathcal{R}(1), C_{13} \cap \mathcal{R}(1), C_{23} \cap \mathcal{R}(2)$

\begin{tabular}{|r||c|c|c|}
\hline Curve & $C_{12} \cap \mathcal{R}(1)$ & $C_{13} \cap \mathcal{R}(1)$ & $C_{23} \cap \mathcal{R}(2)$ \\
\hline \hline Length & 0.9983742022 & 0.2884481319 & 0.02220012557 \\
Density Mass & 2.464527879 & 0.367464849 & 0.036529069 \\
Map $G_{k \ell}$ & $e^{\sqrt{\mathrm{Li}_{2}(x)}-\sqrt{\mathrm{Li}_{2}\left(x^{2}\right)} / 2}$ & $e^{\sqrt{\mathrm{Li}_{2}(x)}-\sqrt{\operatorname{Li}_{2}\left(x^{3}\right) / 3}}$ & $e^{\sqrt{\mathrm{Li}_{2}\left(x^{3}\right)} / 3-\sqrt{\mathrm{Li}_{2}\left(x^{2}\right)} / 2}$ \\
Circular Arc & {$\left[1, G_{12}(T)\right]$} & {$\left[G_{13}(T), G_{13}\left(e^{i \theta_{13}}\right)\right]$} & {$\left[G_{23}(T), G_{23}\left(e^{i \theta_{23}}\right)\right]$} \\
Circular Arc & {$[0,1.996701527]$} & {$[1.388229082,1.755693930]$} & {$[1.077010447,1.113539516]$} \\
Relative Weight & 0.8591630301 & 0.1281025124 & 0.01273445753 \\
\hline
\end{tabular}

where $\left\{c_{n}\right\}$ is an unbounded sequence of increasing positive numbers and $\left\{a_{n}(x)\right\}$ is a sequence of analytic functions that satisfy uniformly on $G^{-1}(S)$ as $n \rightarrow \infty:\left|a_{n}(x)\right| \geq \delta>0, \frac{a_{n}^{\prime}(x)}{n a_{n}(x)}=o(1)$, and $e_{n}(x)=$ $o\left(a_{n}(x) G^{c_{n}}(x)\right)$. Then for any $\varepsilon>0$ all the zeros of $T_{n}(x)$ lie in $C_{\varepsilon}$ for $n$ sufficiently large, where $C_{\varepsilon}$ is the $\varepsilon$-neighborhood of the analytic arc $C$ and

$$
\lim _{n \rightarrow \infty} \frac{1}{c_{n}} \sum\left\{\delta_{G(z)}: z \in Z\left(T_{n}\right) \cap C_{\varepsilon}\right\} \rightarrow \mu
$$

where $\mu$ is normalized Lebesgue measure on the unit circle restricted to the circular arc $G(C)$. In particular, the zero density measure $v_{Z}$ for the family $\left\{T_{n}\right\}$ is $v_{Z}=\mu \circ G$ on the arc $C$.

To apply the Theorem to $\left\{F_{n}(x)\right\}$ requires special normalizations that can be found in our paper [5].

Table 2 gives the essential information about the non-circular boundary curves of the domination regions $\mathcal{R}(1), \mathcal{R}(2)$, and $\mathcal{R}(3)$. The entry "length" means the arc length of the curve while "density mass" means the total measure of the curve with respect to the zero density measure $v_{Z}$. By the Density Theorem, $v_{Z}$ is the pull-back of Lebesgue measure on the unit circle under the conformal mapping $G_{k \ell}$ where $k \ell=12,13$, or 23. The entry "circular arc" means the image of $C_{k \ell}$ under $G_{k \ell}$. Finally by "relative weight", we mean

$$
w_{k, \ell}=v_{Z}\left(C_{k \ell}\right) /\left[v_{Z}\left(C_{12}\right)+v_{Z}\left(C_{13}\right)+v_{Z}\left(C_{23}\right)\right] .
$$

We emphasize that the number of zeros inside the unit disk is determined, of course, by the density measure and not by the length of the curves of the zero attractor. Here is some simple evidence for this. For a particular polynomial $F_{n}$, the number of its zeros near $C_{k \ell}$ is about $w_{k, \ell}$ out of the total number of zeros inside the unit disk in the second quadrant. We confirm this in Table 3. There should be about $w_{13}+w_{23}$ or $14 \%$ of zeros near curves $C_{23}$ and $C_{13}$. At degree 70,000, there are 242 zeros inside the unit disk. About $14 \%$ of them should lie near these two curves or their reflections in the lower half plane. This count yields 17 expected zeros which coincides with the computed number.

In Figure 6, there are plots of zeros along $C_{12}$ and $C_{13}$ of degree 50,000. On each plot the portion of the curve between adjacent boxes have equal zero density measure. This can be understood graphically from counting the number of zeros which are marked by a cross between boxes. The horizontal axes in these plots are arclength. For $C_{12}, s=0$ is the origin; for the other two, $s=0$ is the triple point $T$.

Figure 7 contains the density function for these curves. Since the density function for $C_{12}$ becomes infinite at the origin, we truncated its graph at $s=0.1$. Furthermore, we see that the density functions for $C_{12}$ and $C_{13}$ are lightest in a neighborhood of the triple point $P$ while heaviest near the origin for $C_{12}$ and 

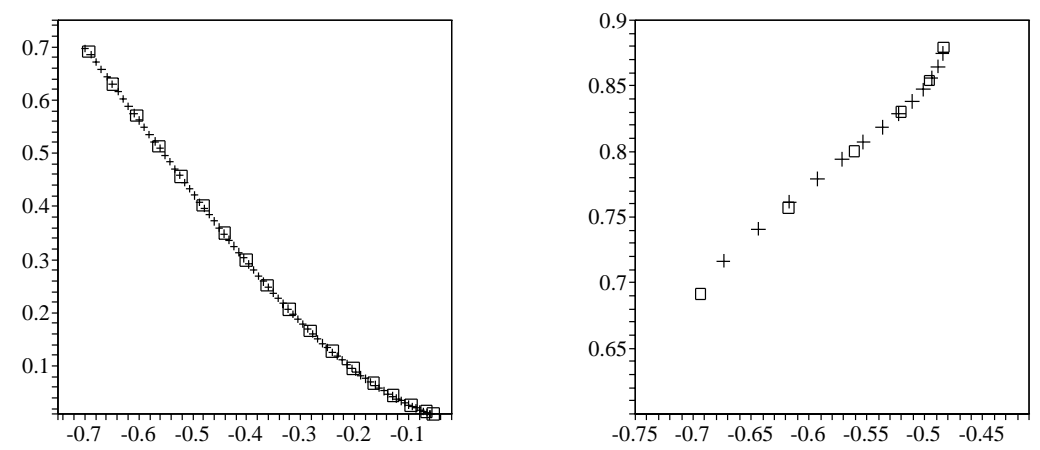

Figure 6: (a) Zeros of Degree 50,000 Along Curve $C_{12}$ and (b) Along Curve $C_{13}$
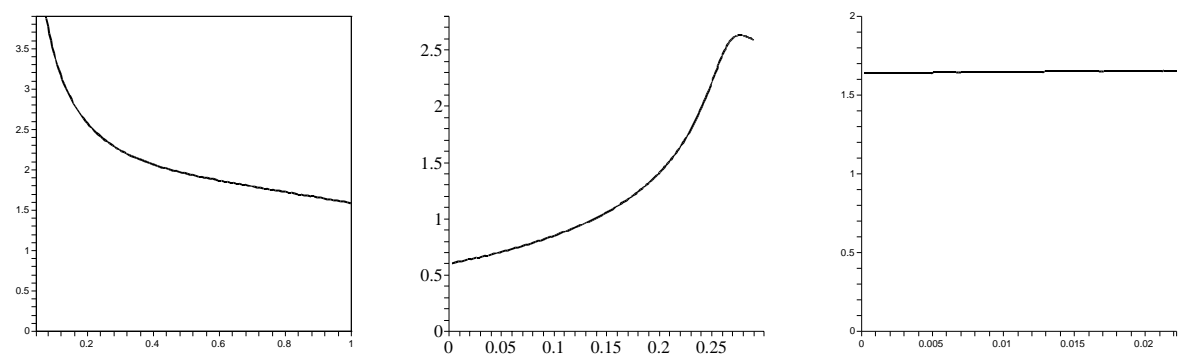

Figure 7: Density Functions as a Function of Arc Length for Curves $C_{12}, C_{13}, C_{23}$

the unit circle at $e^{i \theta_{13}}$ for $C_{13}$. This explains the absence of zeros near the triple point $T$ in Figure 5, even at degree 50,000.

We can also explain why there are so few zeros for the $C_{23}$-family. Its relative weight is 0.0127 . Now this makes two zeros near $C_{23}$ more likely for degree 70,000 over 60,000 yet the opposite occurs. At 70,000, the zero that does occur is nearly at the center of the curve. A second zero would violate the near uniform density. At 60,000, the two zeros that do occur are near the two endpoints which is consistent with the zero density. By equation (1) and the relative weight of $C_{23}$, we do not expect a third zero near this curve until degree 190,000.

In Table 3, the predicted number of zeros near either of the two $\sqrt{\mathrm{Li}_{2}}$-curves $C_{13}$ and $C_{23}$ is found using the $\sqrt{n}$-approximation to the total number of zeros inside the unit disk (see equation $(1)$ ) and the relative weights of these two curves.

Here is another way to get a nearly equivalent prediction of the number of zeros inside the unit disk: $C \cdot \sqrt{n}$ where

$$
C=\frac{v_{Z}\left(C_{12}\right)+v_{Z}\left(C_{13}\right)+v_{Z}\left(C_{23}\right)}{\pi} \simeq 0.9130788466 .
$$

Note that $C$ is remarkably close to the value given by the least squares method in equation (1). 
Table 3: \# Zeros for Combined $C_{13}$ and $C_{23}$ Families

\begin{tabular}{|r||r|r|}
\hline Degree & \# Zeros & Prediction \\
\hline 5000 & 4 & 4.5 \\
10000 & 6 & 6.5 \\
15000 & 8 & 7.9 \\
20000 & 9 & 9.1 \\
25000 & 10 & 10.2 \\
30000 & 11 & 11.2 \\
35000 & 12 & 12.1 \\
40000 & 13 & 12.9 \\
50000 & 14 & 14.5 \\
60000 & 17 & 15.8 \\
70000 & 17 & 17.1 \\
\hline
\end{tabular}

\section{Asymptotics Inside the Unit Disk}

The purpose of this section is to give a flavor of the asymptotics of the partition polynomials needed to confirm their behavior already described. Since the proofs are lengthy, we will be brief.

The generating function for the partition polynomials is a bivariate version of the generating function for the partition numbers which gives an integral version of the polynomials:

$$
P(x, u)=\sum_{n=1}^{\infty} F_{n}(x) u^{n}=\prod_{j=1}^{\infty} \frac{1}{1-x u^{j}}, \quad F_{n}(x)=\frac{1}{2 \pi i} \oint_{|u|=r} \frac{P(x, u)}{u^{n+1}} d u .
$$

We study $F_{n}(x)$ in each of the three regions $\mathcal{R}(k), k=1,2,3$ by using the Circle Method. We begin by writing $F_{n}(x)$ as a contour integral over a circle of radius $r$ :

$$
\begin{aligned}
F_{n}(x) & =\frac{1}{2 \pi i} \oint_{|u|=r} \frac{P(x, u)}{u^{n+1}} d u \\
& =e^{2 \pi n / M} \sum \int_{-\theta_{h, k}^{\prime}}^{\theta_{h, k}^{\prime \prime}} P(x, \exp [2 \pi i(h / k+i z)]) e^{-2 \pi i n h / k} e^{-2 \pi i n \phi} d \phi
\end{aligned}
$$

where the sum is over rational numbers $h / k,\left(\theta_{h, k}^{\prime}, \theta_{h, k}^{\prime \prime}\right)$ are certain subarcs of the circle that contain the point $e^{2 \pi i h / k}$. The choice of the radius $r$ and the order of the denominators $N$ of the fractions $h / k$ requires great care - this is the initial setup of the Circle Method using Farey fractions. See [1], [2], or [7]. Both the radius $r$ and the order of the Farey fractions $N$ depend on the degree of the polynomial $n$ and the point $x$ inside the unit disk, see [5].

The next step is to develop the asymptotics of the integrals $I_{h, k}$

$$
I_{h, k}=\int_{-\theta_{h, k}^{\prime}}^{\theta_{h, k}^{\prime \prime}} P(x, \exp [2 \pi i(h / k+i z)]) e^{-2 \pi i n \phi} d \phi .
$$

This requires a special approximation to the generating function in a neighborhood of the rational points of the unit circle. The proof of this is very lengthy and uses Dirichlet $L$-functions and other techniques from analytic number theory. See our paper [5] for details. 
For relatively prime integers $h<k \leq N$, we introduce the functions $Q_{h, k}(s)$ that come from expanding $\ln \left[P\left(x, e^{2 \pi i h / k+i z}\right)\right]$ and are given in the right plane $\mathfrak{R}(s)>1$ as the double series

$$
Q_{h, k}(s):=\sum_{m \geq 1} \sum_{l \geq 1} \frac{x^{l} e^{2 \pi i l m h / k}}{l}(\operatorname{lm})^{-s} .
$$

We establish that $Q_{h, k}(s)$ has an analytic continuation to $\mathbb{C}$ with a unique simple pole at $s=1$, with residue at $s=1$ is $\frac{1}{k^{2}} \operatorname{Li}_{2}\left(x^{k}\right)$ and its evaluation at 0 is $Q_{h, k}(0)=\frac{1}{2 k} \ln \left(1-x^{k}\right)+\sum_{l(k \nmid l)} \frac{x^{l}}{l} \frac{1}{e^{-2 \pi i l h / k}-1}$.

Theorem 7 Let $h<k$ be relatively prime integers. Fix $|x|<1$, then there is a neighborhood of $u=e^{2 \pi i h / k}$ inside the unit disk where the generating function $P(x, u)$ has the factorization

$$
P\left(x, e^{2 \pi i(h / k+i z)}\right)=e^{w_{h, k}} e^{\Psi_{k}(z)} e^{j_{h, k}(z)}, \quad \Re(z)>0,
$$

where

$$
\begin{aligned}
\Psi_{k}(z) & =\frac{\operatorname{Li}_{2}\left(x^{k}\right)}{2 \pi k^{2}} \frac{1}{z}, \quad j_{h, k}(z)=\frac{1}{2 \pi i} \int_{-\frac{3}{4}+i \infty}^{-\frac{3}{4}+i \infty} Q_{h, k}(s) \Gamma(s)(2 \pi z)^{-s} d s \\
w_{h, k} & =\frac{1}{2 k} \ln \left(1-x^{k}\right)+\sum_{l(k \nmid l)} \frac{x^{l}}{l} \frac{1}{e^{-2 \pi i l h / k}-1}
\end{aligned}
$$

Theorem 7 is crucial step that allows applying the Circle Method to the integrals $I_{h, k}$. Let $f_{\max }(x)=$ $\max \left[f_{1}(x), f_{2}(x), f_{3}(x)\right]$ we state the results:

Theorem 8 (a) For every $k \geq 4$, there exists $\varepsilon \in(0,1)$ so that

$$
\left|I_{h, k}\right| \leq \frac{C}{k} \frac{f_{\max }(x)}{\sqrt{\pi}} \frac{1}{n^{3 / 4}} \exp \left[\sqrt{n}\left((1-\varepsilon) f_{k}(x)+f_{\max }(x)\right)\right]
$$

(b) For $k=1,2$, or 3, on $\overline{\mathcal{R}(k)} \cap U^{+}$:

$$
I_{h, k}=\frac{1}{\sqrt{\pi}} \frac{1}{n^{3 / 4}}\left[\frac{\sqrt{\mathrm{Li}_{2}\left(x^{k}\right)}}{k}\right]^{1 / 2} \exp \left(2 \sqrt{n} \frac{\sqrt{\mathrm{Li}_{2}\left(x^{k}\right)}}{k}\right)\left(1+O\left(\frac{1}{\sqrt{n}}\right)\right)
$$

uniformly on compact subsets.

The above Theorem shows that $I_{0,1}$ is the main contribution to $F_{n}(x)$ on region $\mathcal{R}(1), I_{1,2}$ the main contribution to $F_{n}(x)$ on region $\mathcal{R}(2)$, and $I_{1,3}$ and $I_{2,3}$ are the main contributions to $F_{n}(x)$ on region $\mathcal{R}(3)$. In our last Theorem, we state this precisely. For convenience we introduce the notations $U^{+}$for the upper open unit disk and $I_{k}$ for

$$
I_{k}=\frac{1}{\sqrt{\pi}} \frac{1}{n^{3 / 4}}\left[\frac{\sqrt{\mathrm{Li}_{2}(x)}}{k}\right]^{1 / 2} \exp \left(2 \sqrt{n} \frac{\sqrt{\mathrm{Li}_{2}\left(x^{k}\right)}}{k}\right) .
$$

Theorem 9 (a) In $\overline{\mathcal{R}(1)} \cap U^{+}: F_{n}(x)=e^{w_{0,1}} I_{1}+o_{K_{1}}\left(o\left(I_{1}\right)\right)$ uniformly on compact subsets $K_{1}$.

(b) In $\overline{\mathcal{R}(2)} \cap U^{+}: F_{n}(x)=(-1)^{n} e^{w_{1,2}} I_{2}+o_{K_{2}}\left(o\left(I_{2}\right)\right)$ uniformly on compact subsets $K_{2}$.

(c) In $\overline{\mathcal{R}(3)} \cap U^{+}: F_{n}(x)=\left(e^{-2 \pi i n / 3} e^{w_{1,3}}+e^{-2 \pi i n 2 / 3} e^{w_{2,3}}\right) I_{3}+o_{K_{3}}\left(I_{3}\right)$ uniformly on compact subsets $K_{3}$. 
We can now describe how to normalize the polynomials $F_{n}(x)$ so we can apply the Density Theorem (see Theorem 4).

We can apply Theorem 9 to find the limits $\frac{1}{\sqrt{n}} \ln \left|F_{n}(x)\right|$. For example, on region $\mathcal{R}(1)$,

$$
\begin{aligned}
\frac{1}{\sqrt{n}} \ln \left|F_{n}(x)\right| & =\frac{1}{\sqrt{n}} \ln \left|I_{1}\left(e^{w_{01}}+o(1)\right)\right| \\
& =\frac{1}{\sqrt{n}} \ln \left|\frac{3}{4} \ln n-\ln (2 \pi)+\frac{1}{4} \ln \left[\operatorname{Li}_{2}(x)\right]+2 \sqrt{n} \sqrt{\operatorname{Li}_{2}(x)}\right|+\frac{1}{\sqrt{n}} \ln \left|e^{w_{01}}+o(1)\right| \rightarrow 2 \Re \sqrt{\operatorname{Li}_{2}(x)} .
\end{aligned}
$$

\section{Summary}

We gave an experimental exposition of our work on the zeros of the partition polynomials that are a polynomial version of partition numbers [5]. Intensive computational studies are required to understand their zeros since the number of zeros inside the unit disk is proportional to the square root of their degree. What is especially surprising is that three families of such sparse zeros arise inside the disk. Furthermore, zeros for one of these families, the $C_{23}$ family, do not occur until degree 13,000 and a second zero appears only at degree 60,000 .

Our development of the asymptotics for these polynomials completely determines the limiting behavior of the zeros both as points and their density. This gives an explanation of the rarity of zeros for $C_{23}$.

Among the many polynomial families given by a bivariate infinite product generating function, we single out one related to plane partitions. It would be of great interest to find the zero asymptotics for the polynomial family $\left\{Q_{n}(x)\right\}$ where they are given by the generating function:

$$
\sum_{n=1}^{\infty} Q_{n}(x) u^{n}=\prod_{k=1}^{\infty} \frac{1}{\left(1-x u^{k}\right)^{k}}
$$

We write $Q_{n}(x)=\sum_{m=1}^{n} q_{m}(n) x^{m}$ as usual. By [1], Chapter 11, or [10], the coefficients $q_{m}(n)$ count the number of plane partitions of $n$ whose trace is $m$; that is, the sum of its diagonal entries is $m$. Our last figure is a plot of the zeros of $Q_{100}(x)$.

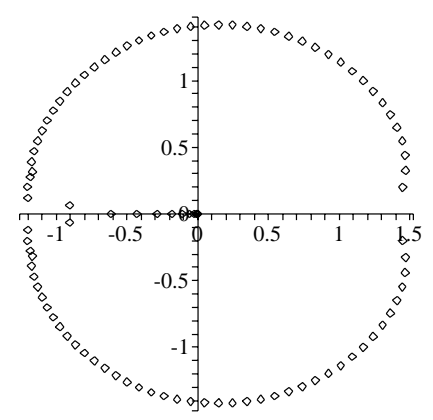

Figure 8: Plane Partition Polynomial Zeros of Degree 100 


\section{References}

[1] George Andrews, The Theory of Partitions, Addison-Wesley, 1976.

[2] Raymond Ayoub, An Introduction to the Analytic Theory of Numbers, AMS Mathematical Surveys 10, 1963.

[3] Dario Bini and G. Fiorentino, Design, Analysis and Implementation of a Multiprecision Polynomial Rootfinder, Numerical Algorithms 23 (2000) 127-173.

[4] Robert P. Boyer and William M. Y. Goh, On the zero attractor of Bernoulli polynomials, Advances in Applied Mathematics 38 (2007) 97-132.

[5] Robert P. Boyer and William M. Y. Goh, The Zero Attractor for Partition Polynomials, Manuscript.

[6] G. H. Hardy and S. Ramanujan, Asymptotic formulae in combinatorial analysis, Proceedings of the London Math. Society (1918) 75-115.

[7] Hans Rademacher, Topics in Analytic Number Theory, Springer-Verlag, 1973.

[8] Edward B. Saff and Vilmos Totik, Logarithmic Potentials with External Fields, Springer Verlag, 1997.

[9] Alan Sokal, Chromatic roots are dense in the whole complex plane, Combinatorics, Probability \& Computing 13 (2004), 221-261.

[10] Richard P. Stanley, The conjugate trace and trace of a plane partition, J. Combinatorial Theory A14 (1973), 53-65.

[11] Richard S. Varga and Amos J. Carpenter, Zeros of the partial sums of $\cos (z)$ and $\sin (z)$, Numerical Algorithms 25 (2000), 363-375.

Department of Mathematics

Drexel University

Philadelphia, PA 19104

email: rboyer at math.drexel.edu 